\title{
Small-time stabilization of homogeneous cascaded systems with application to the unicycle and the slider examples *
}

\author{
Brigitte d’Andréa-Novel $\dagger$ Jean-Michel Coron; Wilfrid Perruquetti ${ }^{\S}$
}

\begin{abstract}
This paper concerns the small-time stabilization of some classes of mechanical systems which are not stabilizable by means of time-invariant continuous state feedback laws. This is the case of nonholonomic systems, an example being the "unicycle-like" mobile robot, or for underactuated mechanical systems, an example being the slider. Explicit time-varying feedback laws leading to small-time stabilization are constructed for these two control systems. The main tools are homogeneity, backstepping, and desingularization technics.
\end{abstract}

Keywords. Nonholonomic kinematic; underactuated mechanical systems; small-time stabilization; homogeneity; time-varying feedback.

\section{Introduction}

We consider nonholonomic kinematics or underactuated mechanical systems for which asymptotic stabilization cannot be achieved through time-invariant continuous state feedback laws since they do not satisfy the necessary condition for feedback stabilization due to Brockett (see 8, [15, Theorem 11.1]; see also [12 for a slightly stronger necessary condition).

In this paper we address the small-time stabilization problem for both systems, and the approach will be illustrated through two examples: the first one is the so-called "unicycle-like" mobile robot which has a nonholonomic kinematic model, and the second one is the "slider" which is an underactuated mechanical system. Our construction of feedback laws stabilizing in small time these two control systems relies on three main ingredients homogeneity, backstepping, and desingularization. Let us stress, that the so-called "unicycle-like" mobile robot covers a large class of mobile robot:1 moving on the 2D-horizontal plane. Indeed, in 9], it is shown that non degenerate mobile robots can be classified into five types, characterized by generic structures of the kinematic model equations. One of these class has the kinematic model of a vertical rolling wheel on the plane (no skidding no sliding). This is the reason why, in robotics, any mobile robot belonging to this class is generically called a "unicycle" mobile robot ${ }^{2}$. For example, a mobile robot having two centered fixed conventional wheel mounted on the same axel and one off-centered steered wheel (not depicted in the figure) as described in Figure 1 is called a unicycle.

Our paper is organized as follows. In Section 2 we briefly recall modeling issues for these two mechanical systems and the similarity between these two kinds of dynamical systems will be emphasized, with respect to controllability and stabilizability. In Section 3 we recall some definitions and give results concerning small-time stabilization and homogeneous control systems, and small-time stabilization of the double integrator. We present our feedback laws stabilizing in small time the unicycle robot in Section 4 and, in Section 5, the slider.

\footnotetext{
${ }^{*}$ This work was supported in part by ANR Finite4SoS (ANR 15 CE23 0007).

${ }^{\dagger}$ MINES ParisTech-CAOR, PSL Research University, 60 Bvd St-Michel, 75006 Paris, brigitte.dandrea-novel@mines-paristech.fr

†Sorbonne Université, Université Paris-Diderot SPC, CNRS, INRIA, Laboratoire Jacques-Louis Lions, LJLL, équipe CAGE, F-75005 Paris, France, coron@ann.jussieu.fr

${ }^{\S}$ CRIStAL (UMR-CNRS 9189), École Centrale de Lille, Citée Scientifique, 59651 Villeneuve-d'Ascq, France, wilfrid.perruquetti@centralelille.fr

${ }^{1}$ This is a rigid cart equipped with wheels.

${ }^{2}$ In the rest of the paper, quotation marks will be omitted: such a model will be simply referred as a unicycle.
} 


\section{Modeling, controllability and stabilizability properties}

In this section the dynamical behaviors of the unicycle robot and the slider are recalled.

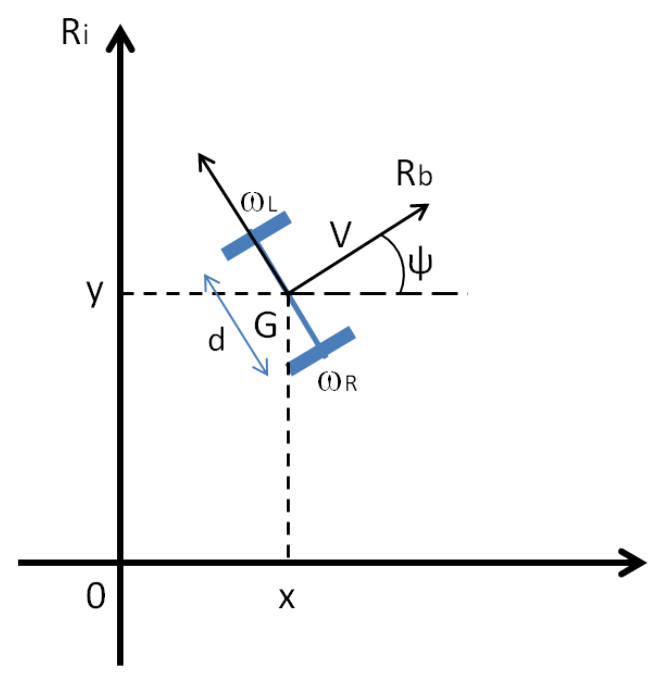

Figure 1: The "unicycle-like" mobile robot

The unicycle robot The unicycle is a classical example of non holonomic vehicle. It moves on the $2 \mathrm{D}$-horizontal plane. The configuration vector $q=(x, y, \psi)^{\top}$ is of dimension $n=3$. The instantaneous unconstrained velocity $\eta=\left(v_{1}, \dot{\psi}\right)^{\top}$ has dimension 2 . It is made of the longitudinal velocity $v_{1}$ and the angular velocity $\dot{\psi}$. The control variables are the angular rotations $\omega_{R}$ and $\omega_{L}$ of the right and left wheels, see Figure 1. The third wheel is a free wheel which does not restrict the robot mobility (for example, an off-centered steered wheel also called a castor wheel). The nonholonomic property of the unicycle comes from the fact that the robot cannot instantaneously move in the lateral direction. Due to the rolling without slipping assumption, the kinematic behavior of point $G$ can be easily obtained from $\omega_{R}, \omega_{L}$ (angular velocities of the right and left wheels), the wheels' radius $R$, and $d$ :

$$
v_{1}=R\left(\omega_{R}+\omega_{L}\right), \Omega=\frac{R}{2 d}\left(\omega_{R}-\omega_{L}\right) .
$$

The kinematic equations of the unicycle robot can then be written as follows.

$$
\dot{x}=v_{1} \cos (\psi), \dot{y}=v_{1} \sin (\psi), \dot{\psi}=\Omega .
$$

Remark 2.1 Let us emphasis, that (2.2) is the simplest nontrivial nonholonomic kinematic system on the plane which corresponds to the kinematic of a vertical rolling wheel (or unicycle and all mobile robots in the class (2,0) see [9]). But many other systems are equivalent to (2.2) such as the Chaplygin sleigh kinematic model (a body that slides frictionlessly on a horizontal plane, with a knife edge that constrains its motion so that the knife slides only longitudinally, see [6, 77]), ... In fact, it is shown in [34] that any kinematic completely nonholonomic system with three states and two control inputs is equivalent to (2.2).

The slider The slider is an underactuated vehicle, similar to marine vehicles (such as hovercrafts or surface vessels), or to terrestrial quadrotors. For more informations about general underactuated vehicles, see, in particular, 22 and the references therein.

The slider moves on a $2 \mathrm{D}$ horizontal plane and has a configuration vector $q$ similar to the unicyle's one: $q=(x, y, \psi)^{\top}$ and $n=3$. But, the instantaneous velocities of vector $\eta$ are not restricted and 


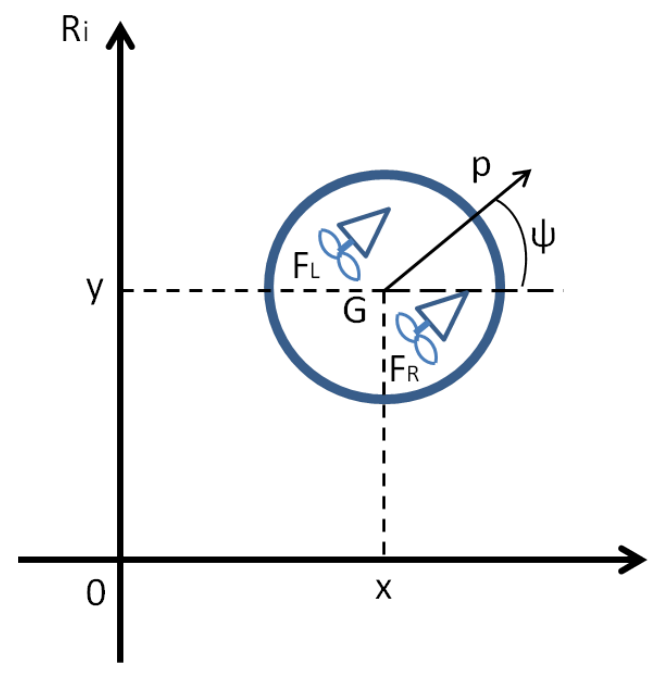

Figure 2: The slider

$\eta$ has dimension 3: $\eta=\left(v_{1}, v_{2}, \dot{\psi}\right)^{\top}$, where $v_{1}$ and $v_{2}$ are respectively the longitudinal and lateral velocities in the robot frame, and $\dot{\psi}$ the angular velocity. Therefore, the control vector has dimension $p=2 \leq n=3$. This vehicle is described in Figure (2). It is actuated by two propellers producing forces $F_{L}$ and $F_{R}$. The sum of these two forces is directly linked to the acceleration of the vehicle, whereas the difference acts on the angular dynamics. Let us denote $\tau_{1}=F_{L}+F_{R}$ and $\tau_{2}=F_{R}-F_{L}$, the dynamics can be written:

$$
\dot{q}=S(q) \eta, m \dot{v}_{1}=m v_{2} \dot{\psi}+\tau_{1}, m \dot{v_{2}}=-m v_{1} \dot{\psi}, I \ddot{\psi}=\tau_{2}
$$

with

$$
S(q)=\left(\begin{array}{ccc}
\cos (\psi) & -\sin (\psi) & 0 \\
\sin (\psi) & \cos (\psi) & 0 \\
0 & 0 & 1
\end{array}\right),
$$

$m$ being the vehicle mass, and $I$ the vehicle inertia. It should be noticed that friction terms have been neglected. Moreover, $S(q)$ is nothing but the rotation matrix from the inertial frame $R_{i}$ to the robot frame $R_{b}$. Therefore, equations (2.3) describe the dynamic behavior of the slider expressed in the vehicle frame and can be written as follows in the inertial frame:

$$
m \ddot{x}=\tau_{1} \cos (\psi), m \ddot{y}=\tau_{1} \sin (\psi), I \ddot{\psi}=\tau_{2} .
$$

The similarity between these equations and the unicycle's ones 2.2 is then obvious.

\subsection{Controllability properties}

The controllability properties of the two systems have been analyzed since a long time. It follows from the Chow-Rashevski theorem (see e.g. [15, Theorem 3.18]) that the unicycle is globally controllable in finite time as well as small-time locally controllable at any equilibrium of the form $(a, 0) \in \mathbb{R}^{3} \times \mathbb{R}^{2}$. The global controllability in small time as well as the small-time local controllability of the slider follows from these two controllability properties of the unicycle. (The small-time local controllability also follows from a general theorem due to Sussmann [48].)

\subsection{Stabilizability properties}

Let us recall that the unicycle control system 2.2 and the slider control system (2.4) cannot be locally asymptotically stabilized by means of time-invariant continuous state feedback laws, as it follows from the following theorem due to Brockett [8]. 
Theorem 2.1 Assume that $f: \mathbb{R}^{n} \times \mathbb{R}^{m} \rightarrow \mathbb{R}^{n}$ is continuous and satisfies $f(0,0)=0$. Assume that there exists a continuous feedback law $u: \mathbb{R}^{n} \rightarrow \mathbb{R}^{m}$ vanishing at $0 \in \mathbb{R}^{n}$ such that $0 \in \mathbb{R}^{n}$ is asymptotically stable for the closed-loop system $\dot{x}=f(x, u(x))$. Then, for any neighborhood $\mathcal{N}$ of $(0,0) \in \mathbb{R}^{n} \times \mathbb{R}^{m}$

$$
f(\mathcal{N}) \text { is a neighborhood of } 0 \in \mathbb{R}^{n} \text {. }
$$

Remark 2.2 The unicycle robot and the slider do not satisfy the Brockett condition (2.5) (and therefore, by Theorem 2.1, are not stabilizable by means of time-invariant continuous state feedback laws). Indeed, for the unicycle, let us consider, as already done in $[8],\left\{e=(0, \epsilon, 0)^{\top}\right\}$ with $\epsilon \neq 0$; it is clear that this element of $\mathbb{R}^{3}$ does not belong to the image of $B \times \mathbb{R}^{2}$ where $B \subset \mathbb{R}^{3}$ is a ball centered at $0 \in \mathbb{R}^{3}$ of radius strictly less than $\pi / 2$ by the unicycle dynamics given by 2.2 . In the same way, for the slider, $\left\{e=(0,0,0, \epsilon, 0,0)^{\top}\right\}$ with $\epsilon \neq 0$ does not belong to the image of $B \times \mathbb{R}^{2}$ by the slider dynamics given by $(5.2)$ below if $B \subset \mathbb{R}^{6}$ is a ball centered at $0 \in \mathbb{R}^{6}$ of radius strictly less than $\pi / 2$.

Consequently, fixed point asymptotic stabilization needs other control approaches than classical time-invariant continuous state feedback laws which allow stabilization of excited reference trajectories. Among those control methods one can cite:

- continuous (with respect to the state) time-varying feedback laws,

- discontinuous feedback laws.

Concerning continuous (with respect to the state) time-varying feedback laws, Coron has shown in 13 that asymptotic stabilization of the origin of a small-time locally controllable driftless affine control system can be achieved through smooth time-periodic state feedback laws (see also [15, Chap. 11, thm. 11.14]). Moreover, this result can be extended to asymptotic stabilization of the origin of many general nonlinear control system using continuous time-varying feedback laws; see [14, 15, Chap. 11, thm. 11.28]. In that context, many authors have proposed stabilizing continuous timevarying feedback laws, see for example [16, 17, 20, 24, 31,39, 45,46 , for the unicycle and related control systems (as chained systems). See also 18, 30, 32 for the stabilization of the attitude of a rigid spacecraft with two controls and [20,29, 35, 36] for stabilization of underactuated surface vessels. Note however that among these articles the only one dealing with finite-time stabilization is [24].

Concerning discontinuous feedback laws, let us mention [1,11 for general results showing that controllable systems can be stabilized by means of discontinuous feedback laws. For robustness issues of discontinuous feedback laws, let us refer to [2, 10, 21, 41, 47. For example of discontinuous feedback laws for the unicycle or related control systems (as chained systems), let us mention 42,43.

In this paper, we are interested in designing small-time stabilizing feedback laws for both systems. Before that, let us briefly recall some results concerning small-time stabilization.

\section{Small-time stabilization}

Let us first introduce some definitions about stabilization and homogeneity.

Definition 3.1 Let $F \in C^{0}\left(\mathbb{R}^{n}, \mathbb{R}^{n}\right)$ be such that $F(0)=0$. The origin $0 \in \mathbb{R}^{n}$ is said to be small-time stable for $\dot{x}=F(x)$ if $\forall \varepsilon>0, \exists \eta>0$ such that

$$
\begin{gathered}
(\dot{x}=F(x) \text { and }|x(0)|<\eta) \Rightarrow(x(\varepsilon)=0), \\
(\dot{x}=F(x) \text { and }|x(0)|<\eta) \Rightarrow(|x(t)|<\varepsilon, \forall t \in[0,+\infty)) .
\end{gathered}
$$

Remark 3.1 Let us point out that, as one can easily check, (3.1) and (3.2) are equivalent to (3.1) and

$$
(\dot{x}=F(x) \text { and } x(0)=0) \Rightarrow(x(t)=0, \forall t \in[0,+\infty)) \text {, }
$$


which is trivially true for system having unique solution which is not assumed here. In this paper, we only assume continuity of ODE's right hand. This assumption ensures existence of solutions (on some time interval) which is an usual assumption when dealing with some stability-like property. Moreover, let us mention that "Small-time stability" is in between the classical concepts of Finitetime stability (see [33, 38]) and Fixed-time stability (see [37, 38]). "Small-time stability" is equivalent to finite-time stability when the settling time function is continuous.

Let $r=\left(r_{1}, \cdots, r_{n}\right)$ be a $n$-tuple of positive real numbers, thereafter called a weight. Then, let us define $\Lambda_{r}:(0,+\infty) \times \mathbb{R}^{n} \rightarrow \mathbb{R}^{n}$ by

$$
\Lambda_{r}(\lambda, x)=\left(\lambda^{r_{1}} x_{1}, \ldots, \lambda^{r_{i}} x_{i}, \ldots, \lambda^{r_{n}} x_{n}\right),
$$

$\forall \lambda \in(0,+\infty), \forall x=\left(x_{1}, \ldots, x_{i}, \ldots, x_{n}\right)^{\top} \in \mathbb{R}^{n}$. This map $\Lambda_{r}$ is called a dilation. Following [26] this map allows to define the notion of functions and vector fields which are $r$-homogeneous .

Definition 3.2 Let $\kappa \in \mathbb{R}$. A function $h: \mathbb{R}^{n} \rightarrow \mathbb{R}$ is said to be $r$-homogeneous of degree $\kappa$ if

$$
h\left(\Lambda_{r}(\lambda, x)\right)=\lambda^{\kappa} h(x), \forall \lambda \in(0,+\infty), \forall x \in \mathbb{R}^{n} .
$$

Definition 3.3 Let $\kappa \in \mathbb{R}$. A vector field $F=\left(F_{1}, \ldots, F_{i}, \ldots, F_{n}\right): \mathbb{R}^{n} \rightarrow \mathbb{R}^{n}$ is said to be $r$ homogeneous of degree $\kappa \in \mathbb{R}$ if, for every $i \in\{1, \ldots, n\}$, the coordinate function $F_{i}$ is $r$-homogeneous of degree $\kappa+r_{i}$.

Among many properties of homogeneous systems, let us recall the following result which will be useful in the sequel.

Theorem 3.1 ( [44]) Let $F$ be a continuous $r$-homogeneous vector-field on $\mathbb{R}^{n}$ of degree $\kappa \in \mathbb{R}$. If $0 \in \mathbb{R}^{n}$ is asymptotically stable for $\dot{x}=F(x)$, then, for every real number $\alpha>\max \left\{r_{1}, \ldots, r_{n}\right\}$, there exists a function $V: \mathbb{R}^{n} \rightarrow \mathbb{R}$ of class $C^{1}$ such that

$$
\begin{gathered}
V \text { is r-homogeneous with degree } \alpha, \\
V(x)>V(0)=0, \forall x \in \mathbb{R}^{n} \backslash\{0\}, \\
\frac{\partial V}{\partial x} \cdot F(x)<0, \forall x \in \mathbb{R}^{n} \backslash\{0\} .
\end{gathered}
$$

Corollary 3.1 ( $[44]$ ) Let $F$ be a continuous $r$-homogeneous vector-field on $\mathbb{R}^{n}$ of degree $\kappa \in$ $(-\infty, 0)$. If $0 \in \mathbb{R}^{n}$ is asymptotically stable for $\dot{x}=F(x)$, then $0 \in \mathbb{R}^{n}$ is small-time stable for $\dot{x}=F(x)$.

Let us now consider the control system

$$
\dot{x}=f(x, u)
$$

where the state is $x \in \mathbb{R}^{n}$, the control is $u \in \mathbb{R}^{m}$, and $f$ is continuous and satisfies $f(0,0)=0$. In the sequel, we intend to design time-piecewise continuous periodic feedback laws for (3.9). Such feedback laws are defined as follows.

Definition 3.4 A function $u: \mathbb{R} \times \mathbb{R}^{n} \rightarrow \mathbb{R}^{l},(t, x) \mapsto u(t, x)$ is a time-piecewise continuous function if there exists a sequence of real numbers $t_{i} \in \mathbb{R}$ such that:

$$
\begin{gathered}
t_{i}<t_{i+1}, \forall i \in \mathbb{Z}, \\
\lim _{i \rightarrow+\infty} t_{i}=+\infty, \\
\lim _{i \rightarrow-\infty} t_{i}=-\infty,
\end{gathered}
$$

$u_{\mid\left(t_{i}, t_{i+1}\right) \times \mathbb{R}^{n}}$ is the restriction to $\left(t_{i}, t_{i+1}\right) \times \mathbb{R}^{n}$ of a continuous function on $\left[t_{i}, t_{i+1}\right] \times \mathbb{R}^{n}$.

Moreover these feedback laws are called time-piecewise continuous time-invariant feedback laws if for $t \in\left(t_{i}, t_{i+1}\right), u(t, x)$ does not depend on $t$. 
From now on, we denote by $C_{\mathrm{t}-\mathrm{p}}\left(\mathbb{R} \times \mathbb{R}^{n} ; \mathbb{R}^{l}\right)$ the set of time-piecewise continuous functions $u(t, x)$ from $\mathbb{R} \times \mathbb{R}^{n}$ to $\mathbb{R}^{l}$.

Let us recall the definition of local asymptotic stability.

Definition 3.5 Let $F \in C_{t-p}\left(\mathbb{R} \times \mathbb{R}^{n} ; \mathbb{R}^{n}\right)$ be such that

$$
F(t, 0)=0, \forall t \in \mathbb{R} .
$$

One says that 0 is locally asymptotically stable for $\dot{x}=F(t, x)$ if

(i) For every $\varepsilon>0$ there exists $\eta>0$ such that, for every $s \in \mathbb{R}$ and for every $\tau \geq s$

$$
(\dot{x}=F(t, x),|x(s)|<\eta) \Rightarrow(|x(\tau)|<\epsilon),
$$

(ii) there exists $\delta>0$ such that for every $\epsilon>0$ there exists $M>0$ such that for every $s \in \mathbb{R}$

$$
(\dot{x}=F(t, x),|x(s)|<\delta) \Rightarrow(|x(\tau)|<\epsilon, \forall \tau \geq s+M) .
$$

Concerning small-time stabilizability by means of time-piecewise continuous periodic feedback laws, we adopt the following definition.

Definition 3.6 The control system (3.9) is locally stabilizable in small time by means of timepiecewise continuous periodic feedback laws of arbitrary small period if, for every positive real number $T$, there exist $\epsilon>0$ and $u \in C_{t-p}\left(\mathbb{R} \times \mathbb{R}^{n} ; \mathbb{R}^{m}\right)$ such that:

$$
\begin{gathered}
u(t, 0)=0, \forall t \in \mathbb{R}, \\
u(t+T, x)=u(t, x), \forall t \in \mathbb{R}, \forall x \in \mathbb{R}^{n}, \\
(\dot{x}=f(x, u(t, x)) \text { and } x(s)=0) \Rightarrow(x(\tau)=0, \forall \tau \geq s), \forall s \in \mathbb{R}, \\
(\dot{x}=f(x, u(t, x)) \text { and }|x(s)| \leq \epsilon) \Rightarrow(x(\tau)=0, \forall \tau \geq s+T), \forall s \in \mathbb{R} .
\end{gathered}
$$

Remark 3.2 If the control $u$ is as in Definition 3.6 then the origin of $\mathbb{R}^{n}$ is locally asymptotically stable for the closed-loop system $\dot{x}=f(x, u(t, x))$.

Before studying the small-time stabilization problem for the unicycle and the slider, let us first prove a result concerning cascaded-systems and let us then apply it first to the small-time stabilization of the double integrator.

\subsection{Small-Time stabilization for homogeneous cascaded systems}

Let us consider the following control system:

$$
\dot{x}=f(x, y), \dot{y}=v,
$$

where the state is $(x, y) \in \mathbb{R}^{n} \times \mathbb{R}$ and the control is $v \in \mathbb{R}$. Let $r=\left(r_{1}, \ldots, r_{n}, r_{n+1}\right)^{\top} \in(0,+\infty)^{n+1}$. For $x=\left(x_{1}, \ldots, x_{n}\right)^{\top} \in \mathbb{R}^{n}$ and for $\lambda \in(0,+\infty)$, we use the notation $\Lambda_{\bar{r}}(\lambda, x)$ introduced in (3.4) with $\bar{r}:=\left(r_{1}, \cdots, r_{n}\right)$. We assume that $f=\left(f_{1}, f_{2}, \ldots, f_{n}\right)$ is of class $C^{0}$ on $\mathbb{R}^{n} \times \mathbb{R}$ and that, for every $i \in\{1, \ldots, n\}, f_{i}$ satisfies the following homogeneity property

$$
f_{i}\left(\Lambda_{\bar{r}}(\lambda, x), \lambda^{r_{n+1}} y\right)=\lambda^{r_{i}+\kappa} f_{i}(x, y),
$$

$\forall x \in \mathbb{R}^{n}, \forall y \in \mathbb{R}, \forall \lambda \in(0,+\infty)$. Let us define $\{x\}^{\{\alpha\}}=\operatorname{sign}(x)|x|^{\alpha}$, for every real number $\alpha>0$ and for every real number $x$. The next theorem is dealing with backstepping and stabilization by means of a continuous feedback law. 
Theorem 3.2 Let us suppose that $\kappa \in \mathbb{R}$ satisfies

$$
\kappa+r_{i}>0, \forall i \in\{1, \ldots, n, n+1\},
$$

and that there exist a continuous feedback law $\bar{y}: \mathbb{R}^{n} \rightarrow \mathbb{R}$ and $l \in(0,+\infty)$ such that

$$
\begin{gathered}
l+1>\frac{r_{i}}{r_{n+1}}, \forall i \in\{1, \ldots, n\}, \\
\bar{y} \text { is } \bar{r} \text {-homogeneous of degree } r_{n+1}, \\
0 \in \mathbb{R}^{n} \text { is asymptotically stable for } \dot{x}=f(x, \bar{y}(x)), \\
\{\bar{y}\}^{\{l\}} \text { is of class } C^{1} .
\end{gathered}
$$

Then the control law

$$
v(x, y)=-k\left\{\{y\}^{\{l\}}-\{\bar{y}(x)\}^{\{l\}}\right\}^{\left\{\left(\kappa+r_{n+1}\right) /\left(l r_{n+1}\right)\right\}},
$$

with a sufficiently high gain $k>0$, makes $0 \in \mathbb{R}^{n+1}$ asymptotically stable for the cascaded system (3.21). Moreover, if $\kappa<0$, this asymptotic stability is a small-time stability.

Remark 3.3 Note that property (3.27) implies that, for every $L \in[l,+\infty)$,

$$
\{\bar{y}\}^{\{L\}} \text { is of class } C^{1} \text {. }
$$

Hence, (3.24) is not an important restriction: it can always been achieved by increasing $l$ if necessary.

Remark 3.4 Theorem 3.2 is related to [30, Proposition 5]. The main improvement of Theorem 3.2 compared to [30, Proposition 5] is that Theorem 3.2 can be applied in a recursive manner, which is not the case of [30, Proposition 5] since, with the notations of this proposition, the feedback law $\left(x_{1}, y, t\right) \in \mathbb{R}^{m} \times \mathbb{R} \times \mathbb{R} \mapsto y-v\left(x_{1}, t\right) \in \mathbb{R}$ is not necessarily of class $C^{1}$ on $\left(\left(\mathbb{R}^{m} \times \mathbb{R}\right) \backslash\{(0,0)\}\right) \times \mathbb{R}$. To apply Theorem 3.2 in a recursive manner, let us first point out that, by Remark 3.3, increasing $l$ if necessary, we may assume that $l \geq 1$. Then $v$ defined by (3.28) is such that

$$
\{v\}^{\left\{\left(l r_{n+1}\right) /\left(\kappa+r_{n+1}\right)\right\}} \text { is of class } C^{1} \text {. }
$$

Let us also point that $v$ satisfies the following homogeneity property

$$
v\left(\Lambda_{\bar{r}}(\lambda, x), \lambda^{r_{n+1}} y\right)=\lambda^{r_{n+1}+\kappa} v(x, y)
$$

$\forall x \in \mathbb{R}^{n}, \forall y \in \mathbb{R}, \forall \lambda \in(0,+\infty)$. Let

$$
r_{n+2}:=r_{n+1}+\kappa
$$

One can then apply Theorem 3.2 to the control system

$$
\dot{x}=f(x, y), \dot{y}=v, \dot{v}=w,
$$

where the state is $(x, y, v) \in \mathbb{R}^{n} \times \mathbb{R} \times \mathbb{R}$ and the control is $w \in \mathbb{R}$, provided that (compare to (3.23))

$$
\begin{gathered}
r_{n+2}+\kappa=r_{n+1}+2 \kappa>0, \\
l+1>\frac{r_{i}}{r_{n+1}+\kappa}, \forall i \in\{1, \ldots, n, n+1\} .
\end{gathered}
$$

One can keep going $k$ times (the state being then in $\mathbb{R}^{n+k+1}$ ) provided that

$$
r_{n+1}+(k+1) \kappa>0
$$

In particular, if $\kappa \geq 0$, one can keep going as long as desired. However in the case where small-time stability is desired (i.e. the case where $\kappa<0)$, condition $(3.36)$ provides an upper bound on $k$. As mentioned in Remark 3.3, the condition on l (see, e.g., (3.35) can always be achieved by increasing $l$. 
Remark 3.5 It has been already proved in [19] that under the assumptions of Theorem 3.2 and even without the assumption (3.27), there exists a continuous feedback law satisfying the homogeneity property (3.31]. Compared to [19] the interest of Theorem 3.2 is that it provides an explicit asymptotically stabilizing feedback law. For $l=1$ Theorem 3.2 is already known: see [30, Prop. 5].

Proof of Theorem 3.2 . Let $\bar{\rho}: \mathbb{R}^{n} \rightarrow[0,+\infty)$ be the following homogeneous "norm" associated to the dilation $\left(x_{1}, \ldots, x_{n}\right)^{\top} \in \mathbb{R}^{n} \rightarrow\left(\lambda^{r_{1}} x_{1}, \ldots, \lambda^{r_{n}} x_{n}\right)^{\top} \in \mathbb{R}^{n}$ :

$$
\bar{\rho}(x)=\sum_{i=1}^{n}\left|x_{i}\right|^{1 / r_{i}}, \forall x=\left(x_{1}, \ldots, x_{n}\right)^{\top} \in \mathbb{R}^{n} .
$$

Let $\alpha \in(0,+\infty)$ be defined by

$$
\alpha:=(l+1) r_{n+1}
$$

Note that, from (3.24, we have

$$
\alpha>r_{i}, \forall i \in\{1, \ldots, n\} .
$$

Using Theorem 3.1 with $r:=\bar{r}$ and $F(x):=f(x, \bar{y}(x))$, the assumptions of Theorem 3.2 and standard homogeneity arguments, one gets the existence of $V \in C^{1}\left(\mathbb{R}^{n}, \mathbb{R}\right)$ and $c_{1} \in(0,1)$ such that

$$
\begin{gathered}
V \text { is } \bar{r} \text {-homogeneous with degree } \alpha>0, \\
c_{1}(\bar{\rho}(x))^{\alpha} \leq V(x) \leq \frac{1}{c_{1}}(\bar{\rho}(x))^{\alpha}, \forall x \in \mathbb{R}^{n}, \\
\frac{\partial V}{\partial x}(x) \cdot f(x, \bar{y}(x)) \leq-2 c_{1}(\bar{\rho}(x))^{\alpha+\kappa}, \forall x \in \mathbb{R}^{n} .
\end{gathered}
$$

Using the "desingularization method" proposed in [40], let us now introduce the following control Lyapunov function candidate for the control system (3.21):

$$
W(x, y)=V(x)+a \Phi(x, y)
$$

with $a$ chosen in $(0,+\infty)$ and

$$
\Phi(x, y):=\int_{\bar{y}(x)}^{y}\left(\{s\}^{\{l\}}-\{\bar{y}(x)\}^{\{l\}}\right) d s=\frac{l}{l+1}|\bar{y}(x)|^{l+1}-y\{\bar{y}(x)\}^{\{l\}}+\frac{|y|^{l+1}}{l+1} .
$$

Let us compute the time-derivative $\dot{W}$ along the trajectories of the closed-loop system

$$
\dot{x}=f(x, y), \dot{y}=-k\left\{\{y\}^{\{l\}}-\{\bar{y}(x)\}^{\{l\}}\right\}^{\left\{\left(\kappa+r_{n+1}\right) /\left(l r_{n+1}\right)\right\}} .
$$

We get

$$
\dot{W}=\dot{V}+a \dot{\Phi}=Q_{1}+Q_{2}+a k Q_{3}+a Q_{4}
$$

with

$$
\begin{gathered}
Q_{1}(x):=\frac{\partial V}{\partial x} f(x, \bar{y}(x)), Q_{2}(x, y):=\frac{\partial V}{\partial x}(f(x, y)-f(x, \bar{y}(x))), \\
Q_{3}(x, y):=-\frac{\partial \Phi}{\partial y}\left\{\{y\}^{\{l\}}-\{\bar{y}(x)\}^{\{l\}}\right\}^{\left\{\left(\kappa+r_{n+1}\right) /\left(l r_{n+1}\right)\right\}}, Q_{4}(x, y):=\frac{\partial \Phi}{\partial x} f(x, y) .
\end{gathered}
$$

Let us point out that $Q_{1}, Q_{2}$, and $Q_{3}$ are well defined and continuous. Noticing that

$$
|\bar{y}(x)|^{l+1}=\left|\{\bar{y}(x)\}^{\{l\}}\right|^{(l+1) / l},
$$

one sees, using also (3.27), that $Q_{4}$ is also well defined and continuous on $\mathbb{R}^{n}$. By 3.42 , we have

$$
Q_{1} \leq-2 c_{1} \bar{\rho}^{\alpha+\kappa}
$$


Let us now deal with $Q_{2}$. For that purpose, let us define, for $p \in \mathbb{N}^{\star},(x, y) \in \mathbb{R}^{n} \times \mathbb{R} \backslash\{(0,0)\}$

$$
G_{p}(x, y)=\frac{\frac{\partial V}{\partial x}(x)(f(x, y)-f(x, \bar{y}(x)))}{c_{1}(\bar{\rho}(x))^{\alpha+\kappa}+p\left|\{y\}^{\{l\}}-\{\bar{y}(x)\}^{\{l\}}\right|^{(\alpha+\kappa) /\left(l r_{n+1}\right)}} .
$$

Let us prove that if $p$ is large enough, we have

$$
\left|G_{p}\right| \leq 1,(x, y) \in \mathbb{R}^{n} \times \mathbb{R} \backslash\{(0,0)\} .
$$

We argue by contradiction, and therefore assume the existence of a sequence $\left(x^{p}, y^{p}\right)_{p \in \mathbb{N}} \in \mathbb{R}^{n} \times \mathbb{R} \backslash$ $\{(0,0)\}$ such that, for $p$ large enough,

$$
\begin{aligned}
c_{1}\left(\bar{\rho}\left(x^{p}\right)\right)^{\alpha+\kappa} & +p\left|\left\{y^{p}\right\}^{\{l\}}-\left\{\bar{y}\left(x^{p}\right)\right\}^{\{l\}}\right|^{(\alpha+\kappa) /\left(l r_{n+1}\right)} \\
& <\left|\frac{\partial V}{\partial x}\left(x^{p}\right)\left(f\left(x^{p}, y^{p}\right)-f\left(x^{p}, \bar{y}\left(x^{p}\right)\right)\right)\right| .
\end{aligned}
$$

Let us point out that $G_{p}$ is $r$-homogeneous with degree 0 . Hence, we may assume that $\rho\left(x^{p}, y^{p}\right)=1$ with

$$
\rho(x, y):=\left|\{y\}^{\{l\}}-\{\bar{y}(x)\}^{\{l\}}\right|^{1 /\left(l r_{n+1}\right)}+\bar{\rho}(x) .
$$

In particular, the sequence $\left(x^{p}, y^{p}\right)_{p \in \mathbb{N}}$ is bounded and, extracting a subsequence if necessary, we may assume that $x^{p} \rightarrow x^{\star}$ and $y^{p} \rightarrow y^{\star}$ as $p \rightarrow+\infty$, with

$$
\rho\left(x^{\star}, y^{\star}\right)=1 \text {. }
$$

Note that by $(3.23)$ and (3.38), $\alpha+\kappa>0$. Hence, using inequality (3.54), we get that $y^{\star}=\bar{y}\left(x^{\star}\right)$ and also that $\bar{\rho}\left(x^{\star}\right)=0$. Therefore, $x^{\star}=0$, and $y^{\star}=0$ which is in contradiction with 3.56). Hence, we can now choose $p=c_{2}$ large enough so that we have:

$$
\left|Q_{2}(x, y)\right| \leq c_{1}(\bar{\rho}(x))^{\alpha+\kappa}+c_{2}\left|\{y\}^{\{l\}}-\{\bar{y}(x)\}^{\{l\}}\right|^{1 /\left(l r_{n+1}\right)}
$$

and it should be noticed that $c_{2}$ does not depend on $a, k$.

Concerning $Q_{3}$ we just point out that, using (3.38), (3.44), and (3.48), we have

$$
Q_{3}(x, y)=-\left|\{y\}^{\{l\}}-\{\bar{y}(x)\}^{\{l\}}\right|^{(\alpha+\kappa) /\left(l r_{n+1}\right)} .
$$

Let us now deal with the last term $Q_{4}$. From (3.44), $\Phi$ is $r$-homogeneous of degree $\alpha$ and therefore $Q_{4}$ is $r$-homogeneous of degree $\alpha+\kappa$. Similarly

$$
(\bar{\rho}(x))^{\alpha+\kappa}+p|y-\bar{y}(x)|^{(\alpha+\kappa) / r_{n+1}}, p>0,
$$

is $r$-homogeneous of degree $\alpha+\kappa$ and is strictly positive outside the origin. Proceeding as for $Q_{2}$, one can show that there exists a sufficiently large positive constant $c_{4}$ such that:

$$
\left|Q_{4}\right| \leq(\bar{\rho}(x))^{\alpha+\kappa}+c_{4}\left|\{y\}^{\{l\}}-\{\bar{y}(x)\}^{\{l\}}\right|^{(\alpha+\kappa) /\left(l r_{n+1}\right)} .
$$

Then, using (3.46), 3.50), (3.57), (3.58), and (3.60), we get

$$
\dot{W} \leq\left(-c_{1}+a\right)(\bar{\rho}(x))^{\alpha+\kappa}+\left(c_{2}+a c_{4}-a k\right)\left|\{y\}^{\{l\}}-\{\bar{y}(x)\}^{\{l\}}\right|^{(\alpha+\kappa) /\left(l r_{n+1}\right)} .
$$

For $a<c_{1}$ and $k$ sufficiently large $\left(k>\left(c_{2}+a c_{4}\right) / a\right)$, we get that $\dot{W}$ is non positive and is equal to zero if and only if $x=0$ and $y=0$ which allows to conclude that the origin of $\mathbb{R}^{n} \times \mathbb{R}$ is asymptotically stable for the closed-loop system (3.45). If $\kappa<0$, this asymptotic stability is a small-time stability due to Corollary 3.1. This concludes the proof of Theorem 3.2.

A natural question arise when $f$, the vector field involved in the cascade form (3.21), is not homogeneous (i.e. does not satisfy the homogeneity property (3.22) but admits a homogeneous approximation $f_{0}$ at the origin. Local homogeneous approximations has been investigated by Massera 28], Rosier [4], Andrieu 3], and recently Efimov [23]. Let $g: \mathbb{R}^{k} \rightarrow \mathbb{R}^{k}$ be a continuous vector field. Let $r=\left(r_{1}, \ldots, r_{k}\right)^{\top} \in(0,+\infty)^{k}$. Let us assume that $g$ has a $r$-homogeneous approximation of at the origin, i.e. that there exists $g_{0}: \mathbb{R}^{k} \rightarrow \mathbb{R}^{k}$ such that for any $x \in S_{r}=\left\{x \in \mathbb{R}^{k}: \sum_{i=1}^{n}\left|x_{i}\right|^{1 / r_{i}}=1\right\}$ we have $\lim _{\lambda \rightarrow 0^{+}} \lambda^{-\kappa} \Lambda_{r}\left(\lambda^{-1}, g\left(\Lambda_{r}(\lambda, x)\right)\right)=g_{0}(x)$ uniformly on $S_{\bar{r}}$ (for some $\kappa \geq-\min _{1 \leq i \leq k} r_{i}, \kappa$ is called the homogeneous degree of $\left.g_{0}\right)$. One has the following theorem 
Theorem 3.3 If $0 \in \mathbb{R}^{k}$ is locally asymptotically stable for $\dot{x}=g_{0}(x)$ then $0 \in \mathbb{R}^{k}$ is locally asymptotically stable for $\dot{x}=g(x)$. Moreover if $\kappa<0$ and if $0 \in \mathbb{R}^{k}$ is small-time stable for $\dot{x}=g_{0}(x)$ then $0 \in \mathbb{R}^{k}$ is small-time stable for $\dot{x}=g(x)$

The asymptotical statement of this theorem is [44, Theorem 3]. The finite-stability statement follows from the proof of [44, Theorem 3] given in [44. As a consequence of this robustness of the small-time stability we have the following corollary.

Corollary 3.2 Let $f_{0}: \mathbb{R}^{n} \times \mathbb{R} \rightarrow \mathbb{R}^{n}$ which satisfies all hypothesis of theorem 3.2: $\kappa \in \mathbb{R}$ satisfies 3.23), there exist $l \in(0,+\infty)$ and a continuous feedback law $\bar{y}: \mathbb{R}^{n} \rightarrow \mathbb{R}$ such that 3.24 3.25 3.26 3.27) holds. Let $f: \mathbb{R}^{n} \times \mathbb{R} \rightarrow \mathbb{R}^{n}$ be a continuous map such that, uniformly on $\Sigma:=\{(x, y) \in$ $\left.\mathbb{R}^{n} \times \mathbb{R} ;|y|^{1 / r_{n+1}}+\sum_{i=1}^{n}\left|x_{i}\right|^{1 / r_{i}}=1\right\}$,

$$
\lim _{\lambda \rightarrow 0^{+}} \lambda^{-\kappa} \Lambda_{\bar{r}}\left(\lambda^{-1}, f\left(\Lambda_{\bar{r}}(\lambda, x), \lambda^{r_{n+1}} y\right)\right)=f_{0}(x, y) .
$$

Then the control law given by (3.28), with a sufficiently high gain $k>0$, makes $0 \in \mathbb{R}^{n+1}$ locally asymptotically stable for the cascaded system 3.21. $f$ ). Moreover, if $\kappa<0$, this local asymptotic stability is a small-time stability.

For the proof of this corollary, it suffices to apply the previously obtained result of [44] to $k:=$ $n+1, \mathbb{R}^{n+1} \simeq \mathbb{R}^{n} \times \mathbb{R}, g(x, y):=(f(x, y), v(x, y))$, and $g_{0}(x, y):=\left(f_{0}(x, y), v(x, y)\right)$. In fact, since our approach also gives homogeneous Lyapunov functions, one can provides a direct proof of

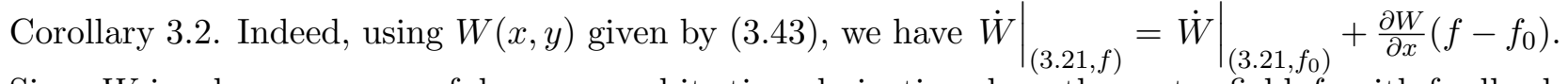
Since $W$ is $r$-homogeneous of degree $\alpha$ and its time derivative along the vector field $f_{0}$ with feedback 3.28 is $r$-homogeneous of degree $\alpha+\kappa$, we have (see 33$)\left.\dot{W}\right|_{\left[3.21 f_{0}\right)} \leq-b_{0} W^{\frac{\alpha+\kappa}{\alpha}},($ see $3.61 p)$ for some positive constant $b_{0}\left(\right.$ take $\left.b_{0}=-\max _{(x, y) \in \Sigma}\left(\frac{\partial W}{\partial x} f_{0}(x, y)+\frac{\partial W}{\partial y} v\right) W^{-(\alpha+\kappa) / \alpha}\right)$. Since $f_{0}$ is the homogeneous approximation at 0 of $f$ in the sense that 3.62 holds and $\frac{\partial W}{\partial x_{i}}$ is $r$-homogeneous (of degree $\left.\alpha-r_{i}\right)$ it implies that $\frac{\partial W}{\partial x}\left(f-f_{0}\right)\left(\Lambda_{\bar{r}}(\lambda, x), \lambda^{r_{n+1}} y\right)=o\left(\lambda^{\alpha+\kappa}\right)$ uniformly on $\Sigma$ as $\lambda \rightarrow 0^{+}$. Thus, for every $(x, y) \in \mathbb{R}^{n} \times \mathbb{R}$ with $|x|+|y|$ small enough, we have:

$$
\left|\frac{\partial W}{\partial x}\left(f-f_{0}\right)\right| \leq \frac{b_{0}}{2} W^{\frac{\alpha+\kappa}{\alpha}}
$$

Hence, for every $(x, y) \in \mathbb{R}^{n} \times \mathbb{R}$ with $|x|+|y|$ small enough,

$$
\left.\dot{W}\right|_{[3.21, f)} \leq-\frac{b_{0}}{2} W^{\frac{\alpha+\kappa}{\alpha}},
$$

which shows that $0 \in \mathbb{R}^{n+1}$ locally asymptotically stable for the cascaded system $\left.3.21, f\right)$. When $\kappa<0$, we have $(\alpha+\kappa) / \alpha<1$ and thus concludes from $(3.63)$ that $0 \in \mathbb{R}^{n+1}$ is small-time stable for this cascaded system $3.21, f)$.

\subsection{Small-time stabilization of the double integrator}

Let us consider the double integrator system:

$$
\dot{x}_{1}=x_{2}, \dot{x}_{2}=u,
$$

where the state is $x=\left(x_{1}, x_{2}\right)^{\top} \in \mathbb{R}^{2}$ and the control is $u \in \mathbb{R}$.

Some results exist concerning the small-time stabilization of system (3.65). One can cite for example [5, 19, 25] for bounded continuous time-invariant small-time stabilizing feedback laws or [4] for an output-feedback control design. These feedback laws are singular when $x_{1}=0$ or $x_{2}=0$, therefore in order to apply "desingularizing functions" as explained in [40] for cascaded system, and similarly in Theorem 3.2 , we refer to the following result inspired by [27] and [4]. 
Theorem 3.4 Let $-\frac{1}{2}<\kappa<0$ and $k_{1}>0, k_{2}>0$ be given. Then $0 \in \mathbb{R}^{2}$ is small-time stable for the double integrator system (3.65) if one uses the following feedback law:

$$
u\left(x_{1}, x_{2}\right)=-k_{1}\left\{x_{1}\right\}^{\{1+2 \kappa\}}-k_{2}\left\{x_{2}\right\}^{\left\{\frac{1+2 \kappa}{1+\kappa}\right\}} .
$$

Remark 3.6 The feedback law (3.66) is a limiting case for [25] which is not covered by [25].

Proof. The time derivative along the solutions of the closed loop system (3.65)- 3.66 of the following Lyapunov function: $V\left(x_{1}, x_{2}\right)=\frac{k_{1}}{2(1+\kappa)}\left|x_{1}\right|^{2(1+\kappa)}+\frac{x_{2}^{2}}{2}$, is $\dot{V}=-k_{2}\left|x_{2}\right|^{\frac{2+3 \kappa}{1+\kappa}} \leq 0$. The LaSalle invariance principle shows that the closed-loop system is globally asymptotically stable. Moreover, the closed-loop system being $(1,1+\kappa)$-homogeneous of negative degree (this degree is $\kappa$ ), the smalltime stability of the origin follows from Corollary 3.1. In fact, instead of using the LaSalle invariance principle, one can construct an homogeneous strict Lyapunov, which is interesting for robustness issues. Indeed, for $\varepsilon>0$, let us consider

$$
V_{\varepsilon}\left(x_{1}, x_{2}\right):=\frac{k_{1}}{2(1+\kappa)}\left|x_{1}\right|^{2(1+\kappa)}+\frac{x_{2}^{2}}{2}+\varepsilon x_{2}\left\{x_{1}\right\}^{\{1+\kappa\}},
$$

which is $(1,1+\kappa)$-homogeneous of degree $2(1+\kappa)$. Then straightforward computations give the existence of an $\varepsilon_{0}>0$ such that, for every $\varepsilon \in\left(0, \varepsilon_{0}\right),(1 / 2) V \leq V_{\varepsilon} \leq 2 V$ and there exists $c>0$ (depending on $\varepsilon$ ) such that $\dot{V}_{\varepsilon} \leq-c V_{\varepsilon}^{\frac{2+3 \kappa}{2(1+\kappa)}}$.

We can also apply our Theorem 3.2 to stabilize in small time $0 \in \mathbb{R}^{2}$ for system (3.65). As already mentioned in Remark 3.4 it gives new feedback laws which are more suitable than the ones given in Theorem 3.4 when one wants to add "integrators", as we are going to do in Section 5 in order to treat the slider control system. Moreover using our proof of Theorem 3.2 we can get precise lower bounds on the gain for the feedback laws as shown in the following theorem (for $\nu=0$ ).

Let us consider the following "modified double integrator" system:

$$
\dot{x}_{1}=\left|x_{1}\right|^{\nu} x_{2}, \dot{x}_{2}=u,
$$

where $0 \leq \nu<1$ and which reduces to 3.65 when $\nu=0$. We have the following theorem.

Theorem 3.5 Let $\nu \in[0,1),-(1-\nu) / 2<\kappa<0, l>1 /(1+\kappa-\nu), k_{1}>0$, and $k_{2}>h\left(l, \kappa, \nu, k_{1}\right)$ be given, where

$$
h\left(l, \kappa, \nu, k_{1}\right):=\frac{2 l(1+\kappa-\nu)^{2}}{(l+1)(1+\kappa-\nu)+\kappa} 2^{\frac{(l-1)((l+1)(1+\kappa-\nu)+\kappa)}{l(1+\kappa-\nu)}} k_{1}^{\frac{1}{1+\kappa-\nu}} .
$$

Then $0 \in \mathbb{R}^{2}$ is small-time stable for the system (3.68) if one uses the following feedback law:

$$
u\left(x_{1}, x_{2}\right)=-k_{2}\left\{\left\{x_{2}\right\}^{\{l\}}-\left\{\bar{x}_{2}\right\}^{\{l\}}\right\}^{\left\{\frac{1+2 \kappa-\nu}{l(1+\kappa-\nu)}\right\}}
$$

where

$$
\bar{x}_{2}=-k_{1}\left\{x_{1}\right\}^{\{1+\kappa-\nu\}} .
$$

Proof. Let us introduce $\left(r_{1}, r_{2}\right)=(1,1+\kappa-\nu) \in(0,+\infty) \times(0,+\infty)$ as the weight of the dilation. Clearly the system (3.68) satisfies $(3.22)$. The proof is a straightforward application of Theorem 3.2 . noticing that the origin is small-time stable for the subsystem:

$$
\dot{x}_{1}=\left|x_{1}\right|^{\nu} \bar{x}_{2}\left(x_{1}\right)=-k_{1}\left\{x_{1}\right\}^{\{1+\kappa\}},
$$

the control law $\bar{x}_{2}\left(x_{1}\right)$ being $r_{1}$-homogeneous of degree $r_{2}$, since $r_{2}=1+\kappa-\nu$. Since $l(1+\kappa-\nu)>1$, $\bar{x}_{2}^{l}\left(x_{1}\right)$ is of class $C^{1}$. Moreover, the vector-field associated to subsystem $x_{1}$ is $\left(r_{1}, r_{2}\right)$-homogeneous of degree $\kappa$. Finally, the closed-loop system (3.65) with the feedback law (3.70) is itself $\left(r_{1}, r_{2}\right)$ homogeneous of degree $\kappa$. We have $\kappa+r_{2}>0$ (because $r_{2}=1+\kappa-\nu$ and $-(1-\nu) / 2<\kappa$ ), so that 
condition (3.23) of Theorem 3.2 holds, meaning that the control law 3.70$)$ is well defined and continuous. Lastly, $\kappa<0$ which implies that $0 \in \mathbb{R}^{2}$ is small-time stable for the modified double integrator system given by (3.68) if $k_{2}>0$ is large enough. In fact, following the proof of Theorem 3.2 one can get the lower bound for $k_{2}$ given in Theorem 3.68 . We set $\alpha=(l+1)(1+\kappa-\nu)>1$ and we consider the Lyapunov function $W\left(x_{1}, x_{2}\right)=\frac{\left|x_{1}\right|^{\alpha}}{\alpha}+a \Phi\left(x_{1}, x_{2}\right)$, where $\Phi\left(x_{1}, x_{2}\right)=\int_{\bar{x}_{2}}^{x_{2}}\left(\{s\}^{\{l\}}-\left\{\bar{x}_{2}\right\}^{\{l\}}\right) d s$. Its time derivative along the solution of the closed-loop system is $\dot{W}=\left\{x_{1}\right\}^{\{\alpha-1\}}\left|x_{1}\right|^{\nu}\left(\bar{x}_{2}+x_{2}-\bar{x}_{2}\right)+a \dot{\Phi}=$ $Q_{1}+Q_{2}+a\left(Q_{3}+Q_{4}\right)$, where $Q_{1}=\left\{x_{1}\right\}^{\{\nu+\alpha-1\}} \bar{x}_{2}=-k_{1}\left|x_{1}\right|^{\alpha+\kappa}, Q_{2}=\left\{x_{1}\right\}^{\{\nu+\alpha-1\}}\left(x_{2}-\bar{x}_{2}\right), Q_{3}=$ $\frac{\partial \Phi}{\partial x_{1}}\left|x_{1}\right|^{\nu} x_{2}=l(1+\kappa-\nu) k_{1}^{l}\left|x_{1}\right|^{2 \nu+\alpha-\kappa-2}\left(x_{2}-\bar{x}_{2}\right) x_{2}, Q_{4}=\frac{\partial \Phi}{\partial x_{2}} u=-k_{2} \mid \varphi\left(x_{1}, x_{2}\right)^{\frac{\alpha+\kappa}{l(1+\kappa-\nu)}}$.

Selecting $a l(1+\kappa-\nu) k_{1}^{1+l}=1$ we get that $\dot{W}=-k_{1}\left|x_{1}\right|^{\alpha+\kappa}-a k_{2} \mid \varphi\left(x_{1}, x_{2}\right)^{\frac{\alpha+\kappa}{l(1+\kappa-\nu)}}+$ $\frac{1}{k_{1}}\left|x_{1}\right|^{\alpha-\kappa-2+2 \nu}\left(\bar{x}_{2}-x_{2}\right)^{2}$. Using Lemma A.1 we get $\left(\bar{x}_{2}-x_{2}\right)^{2} \leq 2^{2 \frac{l-1}{l}}\left|\varphi\left(x_{1}, x_{2}\right)\right|^{\frac{2}{l}}$ and thus

$$
\dot{W} \leq-k_{1}\left|x_{1}\right|^{\alpha+\kappa}-a k_{2}\left|\varphi\left(x_{1}, x_{2}\right)\right|^{\frac{\alpha+\kappa}{l(1+\kappa-\nu)}}+\frac{2^{2 \frac{l-1}{l}}}{k_{1}}\left|x_{1}\right|^{\alpha-\kappa-2+2 \nu}\left|\varphi\left(x_{1}, x_{2}\right)\right|^{\frac{2}{l}} .
$$

Clearly $\dot{W}$ is negative when $\left|x_{1}\right|=0$ and $x_{2} \neq 0$. For $x_{1} \neq 0$ the signum of the right-hand side of this inequality is the same as the signum of $\psi(z)=-k_{1}-a k_{2} z^{\alpha+\kappa}+\frac{2^{2 \frac{l-1}{l}}}{k_{1}} z^{2(1+\kappa-\nu)}$. Lemma A.2. (with $\left.\beta=\alpha+\kappa>\gamma=2(1+\kappa-\nu)>1, a_{0}=k_{1}, a_{1}=a k_{2}, a_{2}=\frac{2^{2 \frac{l-1}{l}}}{k_{1}}\right)$ together with Remark A.1 (from which $(3.69)$ is obtained) we conclude that $\dot{W}<0$ in $\mathbb{R}^{2} \backslash\{0\}$ under the above given conditions for $l, k_{1}, k_{2}, \kappa$ and $\nu$. Finally using Corollary 3.1 , we conclude that $0 \in \mathbb{R}^{2}$ is small-time stable.

Remark 3.7 Theorems 3.4 provides a controller with simple gain selection. However, it does not fit assumptions of Theorem 3.2 and thus cannot be "augmented" to deal with a higher order system by using the "adding integrator technic" as needed in our small time stabilization of the slider. This motivates Theorem 3.5 which is derived from Theorem 3.2. Theorem 3.5 provides precise gain tuning rules whereas Theorem 3.2 just ensures that large enough gains lead to asymptotic stability/small-time stability.

\section{Small-time stabilization of the unicycle robot}

Let us define $\left(x_{1}, x_{2}, x_{3}\right)^{\top} \in \mathbb{R}^{3}$ and $\left(u_{1}, u_{2}\right)^{\top} \in \mathbb{R}^{2}$ by

$$
x_{1}=: x, x_{2}:=y, x_{3}:=\psi, u_{1}:=\nu_{1}, u_{2}:=\Omega .
$$

Then the unicycle control system 2.2 becomes the control system

$$
\dot{x}_{1}=u_{1} \cos \left(x_{3}\right), \dot{x}_{2}=u_{1} \sin \left(x_{3}\right), \dot{x}_{3}=u_{2}
$$

where the state is $\left(x_{1}, x_{2}, x_{3}\right)^{\top} \in \mathbb{R}^{3}$ and the control is $\left(u_{1}, u_{2}\right)^{\top} \in \mathbb{R}^{2}$.

One first observes that if $x_{2}$ and $x_{3}$ vanish at some $t_{0}$ and if, after time $t_{0}, u_{2}$ is equal to 0 , then $x_{2}$ and $x_{3}$ remain equal to 0 . The idea is then to use a first time-invariant feedback law to steer $\left(x_{2}, x_{3}\right)^{\top}$ to $0 \in \mathbb{R}^{2}$ and then take $u_{2}=0$ and use $u_{1}$ to steer $x_{1} \in \mathbb{R}$ to 0 .

Remark 4.1 This strategy is inspired from [14]. In [14] a preliminary time-invariant feedback law is used to steer the control system on (a neighborhood of) a special curve which can be sent to 0 by a special feedback law. This strategy is already used in [24]. The main novelty of our approach compared to 24] is to use this strategy in the framework of homogeneous approximation systems and homogeneous feedback laws. This will allow us to add integrators on the controls and therefore deal with the slider control system 2.4.

We then consider the following quadratic homogeneous approximation of the control system (4.2) (see $[20])$

$$
\dot{x}_{1}=u_{1}, \dot{x}_{2}=u_{1} x_{3}, \dot{x}_{3}=u_{2}
$$


which is a control system where the state is $\left(x_{1}, x_{2}, x_{3}\right)^{\top} \in \mathbb{R}^{3}$ and the control is $\left(u_{1}, u_{2}\right)^{\top} \in \mathbb{R}^{2}$.

As for 4.2), let us observe that, for 4.3), if $x_{2}$ and $x_{3}$ vanish at some $t_{0}$ and if, after time $t_{0}$, $u_{2}$ is equal to 0 , then $x_{2}$ and $x_{3}$ remain equal to 0 . The idea is then, again, to use, for (4.3), a first time-invariant feedback law to steer $\left(x_{2}, x_{3}\right)^{\top}$ to $0 \in \mathbb{R}^{2}$ and then take $u_{2}=0$ and use $u_{1}$ to steer $x_{1} \in \mathbb{R}$ to 0 .

For this homogeneous approximation, a time-piecewise continuous periodic feedback law will be designed leading to the small-time stabilization of (4.3) (Theorem 4.1). Then, using homogeneity arguments, we will check that the same feedback laws leads to the small-time stabilization of the orignal model system (5.2).

In this first design, the control is derived using Theorem 3.5. For this, in a first step, let us choose $u_{1}=\left|x_{2}\right|^{\nu}, \nu \in(0,1)$ and $\bar{x}_{3}=-k_{2}\left\{x_{2}\right\}^{\left\{1+\kappa_{2}-\nu\right\}}, \kappa_{2} \in(-(1-\nu) / 2,0)$, so that the "equivalent" dynamics of the subsystem $x_{2}$ is $\dot{x}_{2}=-k_{2}\left\{x_{2}\right\}^{\left\{1+\kappa_{2}\right\}}$ ( $x_{2}$ converges to zero in small time) and then, the control $u_{2}$ is designed using Theorem 3.5 (a consequence of our cascading result Theorem 3.2) : thus we obtain $x_{2}(t)=x_{3}(t)=0$ for all $t \geq T / 2$ provided that $x_{2}(0)$ and $x_{3}(0)$ are small enough. Note than on this time interval $x_{1}$ increases until $x_{2}$ reaches zero because then $u_{1}=0$ : we should just show that $x_{1}$ remains bounded on a finite time interval. Then, in a second step, note that setting $u_{2}=0$ for $t \in(T / 2, T)$ then $x_{3}, x_{2}$ will remain zero (if they have reached zero). Thus in order to stabilize $x_{1}$ we can choose for $t \in\left(\frac{T}{2}, T\right), u_{1}=-k_{1}\left\{x_{1}\right\}^{\left\{1+\kappa_{1}\right\}},-1<\kappa_{1}<0$. This leads to the following theorem:

Theorem 4.1 Let $T>0, \nu \in(0,1),-1<\kappa_{1}<0,-(1-\nu) / 2<\kappa_{2}<0, l>1 /\left(1+\kappa_{2}-\nu\right), k_{1}>0$, and $k_{2}>0$. Then, for every $k_{3}>h\left(l, \kappa_{2}, \nu, k_{2}\right)$ (where the function $h$ is given by (3.69)) for the feedback law $u=\left(u_{1}, u_{2}\right) \in C_{t-p}\left(\mathbb{R} \times \mathbb{R}^{3} ; \mathbb{R}^{2}\right)$ defined by

$$
\begin{gathered}
u_{1}=\left|x_{2}\right|^{\nu} \text { in }\left[0, \frac{T}{2}\right] \times \mathbb{R}^{3}, \\
u_{2}=-k_{3}\left\{\left\{x_{3}\right\}^{\{l\}}-\left\{\bar{x}_{3}\right\}^{\{l\}}\right\}^{\left\{\frac{1+2 \kappa_{2}-\nu}{l\left(1+\kappa_{2}-\nu\right)}\right\}} \text { in }\left[0, \frac{T}{2}\right] \times \mathbb{R}^{3}, \text { with } \bar{x}_{3}=-k_{2}\left\{x_{2}\right\}^{\left\{1+\kappa_{2}-\nu\right\}}, \\
u_{1}=-k_{1}\left\{x_{1}\right\}^{\left\{1+\kappa_{1}\right\}}, \text { in }\left(\frac{T}{2}, T\right) \times \mathbb{R}^{3}, \\
u_{2}=0 \text { in }\left(\frac{T}{2}, T\right) \times \mathbb{R}^{3}, \\
u(t+T, x)=u(t, x), \forall t \in \mathbb{R}, \forall x \in \mathbb{R}^{3},
\end{gathered}
$$

and for $f(x, u)=\left(u_{1}, u_{1} x_{3}, u_{2}\right)^{\top}$, (3.17), (3.18), and (3.19) hold and there exists $\epsilon>0$ such that (3.20) holds too. In particular (4.3) is small-time locally stabilizable by means of explicit time-varying piecewise continuous time-invariant feedback laws.

Proof. On $[0, T / 2]$, the closed-loop system is

$$
\left\{\begin{array}{l}
\dot{x}_{1}=\left|x_{2}\right|^{\nu}, \\
\dot{x}_{2}=\left|x_{2}\right|^{\nu} x_{3}, \\
\dot{x}_{3}=-k_{3}\left\{\left\{x_{3}\right\}^{\{l\}}-\left\{\bar{x}_{3}\right\}^{\{l\}}\right\}^{\left\{\frac{1+2 \kappa_{2}-\nu}{l\left(1+\kappa_{2}-\nu\right)}\right\}} .
\end{array}\right.
$$

We apply the proof of Theorem 3.5 to the $\left(x_{2}, x_{3}\right)$-subsystem. We set $\alpha=(l+1)\left(1+\kappa_{2}-\nu\right)$ and we consider the Lyapunov function $W\left(x_{2}, x_{3}\right)=\frac{\left|x_{2}\right|^{\alpha}}{\alpha}+a \Phi\left(x_{2}, x_{3}\right)$, where $\Phi\left(x_{2}, x_{3}\right)=\int_{\bar{x}_{3}}^{x_{3}}\left(\{s\}^{\{l\}}-\right.$ $\left.\left\{\bar{x}_{3}\right\}^{\{l\}}\right) d s$ and $a$ is such that $a l\left(1+\kappa_{2}-\nu\right) k_{2}^{1+l}=1$. From the proof of Theorem 3.5 we know that

$$
\dot{W} \leq-\frac{1}{C} W^{\frac{\alpha+\kappa_{2}}{\alpha}} .
$$

In 4.9 and in the following $C$ denotes various positive constant which may vary from line to line but are independent of $x$. We also have on $[0, T / 2]$

$$
\left|\dot{x}_{1}\right| \leq C W^{\frac{\nu}{\alpha}}
$$


On $(T / 2, T)$ the closed-loop system is

$$
\dot{x}_{1}=-k_{1}\left\{x_{1}\right\}^{1+\kappa_{1}}, \dot{x}_{2}=-k_{1}\left\{x_{1}\right\}^{1+\kappa_{1}} x_{3}, \dot{x}_{3}=0 .
$$

In particular, if $V\left(x_{1}\right):=x_{1}^{2}$,

$$
\dot{V} \leq-\frac{1}{C} V^{\frac{2+\kappa_{1}}{2}}
$$

We also have on $(T / 2, T)$

$$
\left|\dot{x}_{2}\right|+\left|\dot{x}_{3}\right| \leq C\left(\left|x_{2}\right|+\left|x_{3}\right|\right) V^{\frac{1+\kappa_{1}}{2}} .
$$

The conclusion of Theorem 4.1 easily follows from (4.9), 4.10), 4.12), and 4.13). (Let us recall that $\kappa_{1}<0$ and $\kappa_{2}<0$.)

Let us now return to the initial control system (4.2). Let us deduce from Theorem 4.1 the following theorem.

Theorem 4.2 Let $T>0, \nu \in(0,1),-1<\kappa_{1}<0,-(1-\nu) / 2<\kappa_{2}<0, k_{1}>0$, and $k_{2}>0$. Then there exists a large enough $k_{3}>0$ such that, for the feedback law $u=\left(u_{1}, u_{2}\right) \in C_{t-p}\left(\mathbb{R} \times \mathbb{R}^{3} ; \mathbb{R}^{2}\right)$ defined by (4.4) to (4.7) and for $f(x, u)=\left(u_{1} \cos \left(x_{3}\right), u_{1} \sin \left(x_{3}\right), u_{2}\right)^{\top}$, (3.17), (3.18), and (3.19) hold and there exists $\epsilon>0$ such that 3.20 holds too. In particular 4.2 is locally stabilizable in small time by means of explicit time-piecewise continuous time-invariant feedback laws.

Proof. The proof is a direct consequence of Corollary 3.2 combined with the proof given above for Theorem 4.1. One can also proceed in a more direct way. Indeed simple computations and estimates show that (4.9), 4.10), 4.12), and (4.13) still hold for (4.2) at least if $\left|x_{1}\right|+\left|x_{2}\right|+\left|x_{3}\right|$ is small enough.
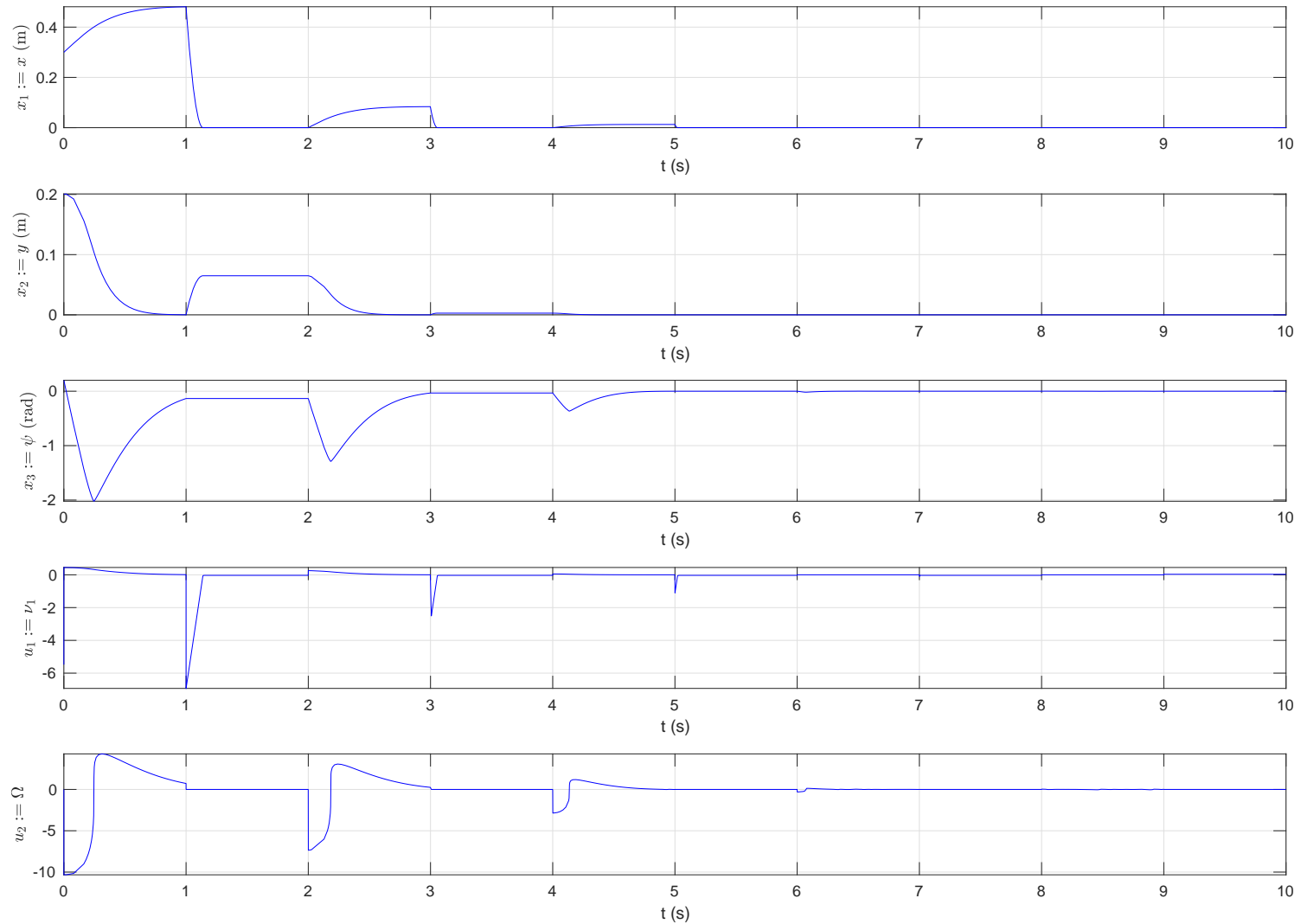

Figure 3: Simulation of 4.2 using control 4.7) with $T=2, \nu=0.5 ; \kappa_{1}=-0.5, \kappa_{2}=-0.1, l=$ $3, k_{1}=10, k_{2}=k_{3}=5$. 


\section{Small-time stabilization of the slider}

Let us define $\left(x_{1}, x_{2}, x_{3}, x_{4}, x_{5}, x_{6}\right)^{\top} \in \mathbb{R}^{6}$ and $\left(u_{1}, u_{2}\right)^{\top} \in \mathbb{R}^{2}$ by

$$
x_{1}=: x, x_{2}:=\dot{x}, x_{3}:=y, x_{4}:=\dot{y}, x_{5}:=\psi, x_{6}:=\dot{x}_{5}, u_{1}:=\frac{\tau_{1}}{m}, u_{2}:=\frac{\tau_{2}}{I} .
$$

Then the slider control system (2.4) becomes the control system

$$
\dot{x}_{1}=x_{2}, \dot{x}_{2}=u_{1} \cos \left(x_{5}\right), \dot{x}_{3}=x_{4}, \dot{x}_{4}=u_{1} \sin \left(x_{5}\right), \dot{x}_{5}=x_{6}, \dot{x}_{6}=u_{2},
$$

where the state is $x=\left(x_{1}, x_{2}, x_{3}, x_{4}, x_{5}, x_{6}\right)^{\top} \in \mathbb{R}^{6}$ and the control is $\left(u_{1}, u_{2}\right)^{\top} \in \mathbb{R}^{2}$. Similarly to the unicycle, one first observes that if $x_{3}\left(t_{0}\right)=x_{4}\left(t_{0}\right)=x_{5}\left(t_{0}\right)=x_{6}\left(t_{0}\right)=0$ and if, after time $t_{0}, u_{2}$ is equal to 0 , then $x_{3}(t)=x_{4}(t)=x_{5}(t)=x_{6}(t)=0$. The idea is then to use a first timeinvariant feedback law to steer $\left(x_{3}, x_{4}, x_{5}, x_{6}\right)^{\top}$ to $0 \in \mathbb{R}^{4}$ and then take $u_{2}=0$ and use $u_{1}$ to steer $\left(x_{1}, x_{2}\right)^{\top} \in \mathbb{R}$ to $0 \in \mathbb{R}^{2}$.

As for the unicycle, let us consider the following quadratic approximation of 5.2 ) (see, once more, 20 )

$$
\dot{x}_{1}=x_{2}, \dot{x}_{2}=u_{1}, \dot{x}_{3}=x_{4}, \dot{x}_{4}=u_{1} x_{5}, \dot{x}_{5}=x_{6}, \dot{x}_{6}=u_{2},
$$

where the state is still $x=\left(x_{1}, x_{2}, x_{3}, x_{4}, x_{5}, x_{6}\right)^{\top} \in \mathbb{R}^{6}$ and the control is still $\left(u_{1}, u_{2}\right)^{\top} \in \mathbb{R}^{2}$.

As for (5.2), we have for (5.3) that if $x_{3}\left(t_{0}\right)=x_{4}\left(t_{0}\right)=x_{5}\left(t_{0}\right)=x_{6}\left(t_{0}\right)=0$ and if, after time $t_{0}, u_{2}$ is equal to 0 , then $x_{3}(t)=x_{4}(t)=x_{5}(t)=x_{6}(t)=0$. Let us therefore choose, according to Theorem 3.2 the controls $u_{1}$ and $u_{2}$ in a first step, so that the dynamics of the subsystem $\left(x_{3}, x_{4}, x_{5}, x_{6}\right)^{\top}$ is such that we obtain $x_{3}(t)=x_{4}(t)=x_{5}(t)=x_{6}(t)=0$ for all $t \geq T / 2$ provided that $x_{3}(0), x_{4}(0)$, $x_{5}(0)$ and $x_{6}(0)$ are small enough. Then we impose $u_{2}=0$ for $t \geq T / 2, x_{3}, x_{4}, x_{5}$ and $x_{6}$ will remain zero. To stabilize in small time the double integrator made of the state variables $x_{1}$ and $x_{2}$, we can choose for $t>T / 2, u_{1}$ as in 3.70 with $\nu=0$.

Let $k_{1}>0, k_{2}>0, k_{3}>0, k_{4}>0, k_{5}>0, k_{6}>0, \kappa_{1} \in(-1 / 2,0)$, and $\mu \in(0,1)$. We assume that

$$
\begin{aligned}
& -\frac{1-\mu}{2(2-\mu)}<\kappa_{2}<0 \\
& r_{1}:=1, r_{2}:=1+\kappa_{1}, r_{3}:=1, r_{4}:=r_{3}+\kappa_{2}, \\
& r_{5}:=\left(r_{4}+\kappa_{2}\right)(1-\mu), r_{6}:=r_{5}+\kappa_{2}, r_{7}:=r_{6}+\kappa_{2} \text {, } \\
& \bar{x}_{4}:=-k_{3}\left\{x_{3}\right\}^{\left\{\frac{r_{4}}{r_{3}}\right\}}, \\
& \bar{x}_{5}:=-k_{4}\left\{\left\{x_{4}\right\}^{\left\{\frac{r_{3}}{r_{4}}\right\}}-\left\{\bar{x}_{4}\right\}^{\left\{\frac{r_{3}}{r_{4}}\right\}}\right\}^{\left\{\frac{r_{5}}{r_{3}}\right\}}, \\
& \bar{x}_{6}:=-k_{5}\left\{\left\{x_{5}\right\}^{\left\{\frac{r_{3}}{r_{5}}\right\}}-\left\{\bar{x}_{5}\right\}^{\left\{\frac{r_{3}}{r_{5}}\right\}}\right\}^{\left\{\frac{r_{6}}{r_{3}}\right\}} \text {. }
\end{aligned}
$$

Note that (5.4) insures that $r_{4}>0, r_{5}>0, r_{6}>0$, and $r_{7}>0$. Let us then prove the following theorem, ensuring the small-time stabilization of the origin for (5.3) with explicit time-varying feedback laws.

Theorem 5.1 Let $T>0, k_{1}>0, k_{2}>0, k_{3}>0, k_{4}>0, \kappa_{1} \in(-1 / 2,0), \mu \in(0,1)$ and $\kappa_{2}<0$ be given so that (5.4) is satisfied. We assume that

$$
k_{4}>h\left(\frac{1}{1+\kappa_{2}}, \kappa_{2}, 0, k_{3}\right)=2^{-2 \kappa_{2}} k_{3}^{\frac{1}{1+\kappa_{2}}}
$$

(let us recall that $h$ is defined in (3.69). Then there exists $K_{5}>0$ such that, for every $k_{5} \geq K_{5}$, there 
exists $K_{6}$ such that, for every $k_{6} \geq K_{6}$, the following feedback law $u=\left(u_{1}, u_{2}\right) \in C_{t-p}\left(\mathbb{R} \times \mathbb{R}^{6} ; \mathbb{R}^{2}\right)$ :

$$
\begin{gathered}
u_{1}=\left|\left\{x_{4}\right\}^{\left\{\frac{r_{3}}{r_{4}}\right\}}-\left\{\bar{x}_{4}\right\}^{\left\{\frac{r_{3}}{r_{4}}\right\}}\right|^{\frac{\mu r_{5}}{r_{3}}} \text { in }[0, T / 2] \times \mathbb{R}^{6}, \\
u_{2}=-k_{6}\left\{\left\{x_{6}\right\}^{\left\{\frac{r_{3}}{r_{6}}\right\}}-\left\{\bar{x}_{6}\right\}^{\left\{\frac{r_{3}}{r_{6}}\right\}}\right\}^{\left\{\frac{r_{6}+\kappa_{2}}{r_{3}}\right\}} \text { in }[0, T / 2] \times \mathbb{R}^{6}, \\
u_{1}=-k_{1}\left\{x_{1}\right\}^{\left\{\frac{r_{2}+\kappa_{1}}{r_{1}}\right\}}-k_{2}\left\{x_{2}\right\}^{\left\{\frac{r_{2}+\kappa_{1}}{r_{2}}\right\}} \text { in }(T / 2, T) \times \mathbb{R}^{6}, \\
u_{2}=0 \text { in }(T / 2, T) \times \mathbb{R}^{6}, \\
u(t+T, x)=u(t, x), \forall t \in \mathbb{R}, \forall x \in \mathbb{R}^{6},
\end{gathered}
$$

is such that, for the homogeneous approximation (5.3) and for the slider control system (5.2), (3.17), (3.18), and (3.19) hold, and there exists $\epsilon>0$ such that, for

$$
f(x, u)=\left(x_{2}, u_{1}, x_{4}, u_{1} x_{5}, x_{6}, u_{2}\right)^{\top}
$$

and for

$$
f(x, u)=\left(x_{2}, u_{1} \cos \left(x_{5}\right), x_{4}, u_{1} \sin \left(x_{5}\right), x_{6}, u_{2}\right)^{\top},
$$

one has

$$
(\dot{x}=f(x, u(t, x)) \text { and }|x(s)| \leq \epsilon) \Rightarrow(x(\tau)=0), \forall \tau \geq s+2 T), \forall s \in \mathbb{R} .
$$

In particular (5.2) is locally stabilizable in small time by means of explicit time-piecewise continuous time-invariant feedback laws.

Proof of Theorem 5.1. The proof is an application of Theorem 3.2, Theorem 3.3. Theorem 3.4 and Theorem 3.5 with $\nu=0$. Let us first deal with (5.3). On $[0, T / 2]$, the quadratic approximation (5.3) reads as

$$
\left\{\begin{array}{l}
\dot{x}_{3}=x_{4}, \\
\dot{x}_{4}=\mid\left\{x_{4}\right\}^{\left\{\frac{r_{3}}{r_{4}}\right\}}-\left\{\bar{x}_{4}\right\}^{\left.\left\{\frac{r_{3}}{r_{4}}\right\}\right|^{\frac{\mu r_{5}}{r_{3}}} x_{5},} \\
\dot{x}_{5}=x_{6}, \\
\dot{x}_{6}=-k_{6}\left\{\left\{x_{6}\right\}^{\left\{\frac{r_{5}}{r_{6}}\right\}}-\left\{\bar{x}_{6}\right\}^{\left\{\frac{r_{5}}{r_{6}}\right\}}\right\}^{\left\{\frac{r_{6}+\kappa_{2}}{r_{5}}\right\}} .
\end{array}\right.
$$

Let us consider the control system

$$
\dot{x}_{3}=x_{4}, \dot{x}_{4}=\left|\left\{x_{4}\right\}^{\left\{\frac{r_{3}}{r_{4}}\right\}}-\left\{\bar{x}_{4}\right\}^{\left\{\frac{r_{3}}{r_{4}}\right.}\right|^{\frac{\mu r_{5}}{r_{3}}} x_{5},
$$

where the state is $\left(x_{3}, x_{4}\right)^{\top} \in \mathbb{R}^{2}$ and the control is $x_{5} \in \mathbb{R}$. It follows from Theorem 3.5 with $\nu=0$ and (5.10) that $0 \in \mathbb{R}^{2}$ is small-time stable for this control system if one uses the feedback law $x_{5}=\bar{x}_{5}$. Let us point out that if

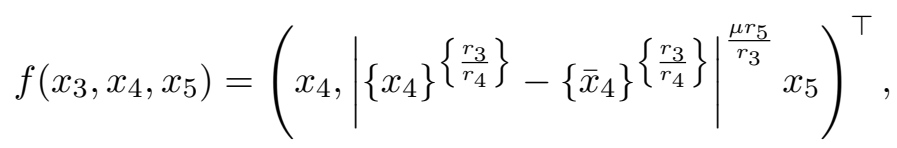

one has

$$
\begin{aligned}
& f_{1}\left(\lambda^{r_{3}} x_{3}, \lambda^{r_{4}} x_{4}, \lambda^{r_{5}} x_{5}\right)=\lambda^{r_{3}+\kappa_{2}} f_{1}\left(x_{3}, x_{4}, x_{5}\right), \\
& f_{2}\left(\lambda^{r_{3}} x_{3}, \lambda^{r_{4}} x_{4}, \lambda^{r_{5}} x_{5}\right)=\lambda^{r_{4}+\kappa_{2}} f_{2}\left(x_{3}, x_{4}, x_{5}\right) .
\end{aligned}
$$


Moreover, for $l:=r_{3} / r_{6}$, one has, using (5.4, 5.5) and (5.6),

$$
\begin{gathered}
l+1>\frac{r_{3}}{r_{6}}>\frac{r_{4}}{r_{6}}>\frac{r_{5}}{r_{6}}>0, \\
\bar{x}_{5}\left(\lambda^{r_{3}} x_{3}, \lambda^{r_{4}} x_{4}\right)=\lambda^{r_{5}} \bar{x}_{5}\left(x_{3}, x_{4}\right), \\
\left\{\bar{x}_{5}\right\}^{\{l\}} \text { is of class } C^{1} .
\end{gathered}
$$

Then, by Theorem 3.2 , at least if $k_{5}>0$ is large enough, $0 \in \mathbb{R}^{3}$ is small-time stable for

$$
\left\{\begin{array}{l}
\dot{x}_{3}=x_{4}, \\
\dot{x}_{4}=\mid\left\{x_{4}\right\}^{\left\{\frac{r_{3}}{r_{4}}\right\}}-\left\{\bar{x}_{4}\right\}^{\left.\left\{\frac{r_{3}}{r_{4}}\right\}\right|^{\frac{\mu r_{5}}{r_{3}}} x_{5},} \\
\dot{x}_{5}=-k_{5}\left\{\left\{x_{5}\right\}^{\left\{\frac{r_{3}}{r_{5}}\right\}}-\left\{\bar{x}_{5}\right\}^{\left\{\frac{r_{3}}{r_{5}}\right\}}\right\}^{\left\{\frac{r_{6}}{r_{3}}\right\}}=\bar{x}_{6} .
\end{array}\right.
$$

Note that $\left\{\bar{x}_{6}\right\}^{\left\{l^{\prime}\right\}}$ is of class $C^{1}$ for $l^{\prime}:=r_{3} / r_{7}$. One applies once more Theorem 3.2 (see also Remark 3.4). Using once more (5.4), (5.5) and (5.6), one gets

$$
\begin{aligned}
& r_{7}=r_{6}+\kappa_{2}>0, \\
& l+1=\frac{r_{3}}{r_{6}}+1>\frac{r_{3}}{r_{6}+\kappa_{2}}>\frac{r_{4}}{r_{6}+\kappa_{2}}>\frac{r_{5}}{r_{6}+\kappa_{2}}>\frac{r_{6}}{r_{6}+\kappa_{2}}>0 .
\end{aligned}
$$

One gets that, under the assumptions of Theorem 5.1, $0 \in \mathbb{R}^{4}$ is small-time stable for (5.27). By Theorem 3.3 one gets that, under the same assumptions, $0 \in \mathbb{R}^{4}$ is small-time stable for

$$
\left\{\begin{array}{l}
\dot{x}_{3}=x_{4}, \\
\dot{x}_{4}=\mid\left\{x_{4}\right\}^{\left\{\frac{r_{3}}{r_{4}}\right\}}-\left\{\bar{x}_{4}\right\}^{\left\{\frac{r_{3}}{r_{4}}\right\}^{\frac{\mu r_{5}}{r_{3}}} \sin \left(x_{5}\right),} \\
\dot{x}_{5}=-k_{5}\left\{\left\{x_{5}\right\}^{\left\{\frac{r_{3}}{r_{5}}\right\}}-\left\{\bar{x}_{5}\right\}^{\left\{\frac{r_{3}}{r_{5}}\right\}}\right\}^{\left\{\frac{r_{6}}{r_{3}}\right\}}=\bar{x}_{6} .
\end{array}\right.
$$

In particular if $x:[0, T / 2] \rightarrow \mathbb{R}^{6}$ is a solution of the closed-loop systems $\dot{x}=f(x, u(t, x))$ where $f$ is given by (5.16) or by (5.17), we have $x_{3}(T / 2)=x_{4}(T / 2)=x_{5}(T / 2)=x_{6}(T / 2)=0$ if $\left|x_{3}(0)\right|+$ $\left|x_{4}(0)\right|+\left|x_{5}(0)\right|+\left|x_{6}(0)\right|$ is small enough.

On $[T / 2, T]$, one has, if $x_{5}(T / 2)=x_{6}(T / 2)=0$ for $f$ given by (5.17) and for every $\left(x_{5}(T / 2), x_{6}(T / 2)\right)$ for $f$ given by $(5.16)$,

$$
\dot{x}_{1}=x_{2}, \dot{x}_{2}=-k_{1}\left\{x_{1}\right\}^{\left\{\frac{r_{2}+\kappa_{1}}{r_{1}}\right\}}-k_{2}\left\{x_{2}\right\}^{\left\{\frac{r_{2}+\kappa_{1}}{r_{2}}\right\}} .
$$

Note that, by Theorem $3.4,0 \in \mathbb{R}^{2}$ is small-time stable for 5.31). Theorem 5.1 follows from the above arguments.

Remark 5.1 In Theorem 5.1, for $t \in(T / 2, T)$ one can replace $u_{1}$ defined in (5.13) by $u_{1}=u$ where $u$ is defined in (3.70) provided that $l, \kappa, k_{1}$, and $k_{2}$ satisfy the assumptions of Theorem 3.5 with $\nu=0$. Indeed it suffices to then use in the above proof Theorem 3.5 with $\nu=0$ instead of Theorem 3.4 .

\section{Conclusion}

To conclude, new explicit feedback laws, based on cascaded and desingularization techniques, and homogeneity properties, have been proposed leading to small-time stabilization of two classical examples of nonholonomic or underactuated mechanical systems: the unicycle robot and the slider, which are known to be not asymptotically stabilizable by means of continuous feedback laws. The proposed resulting feedback laws have been first established considering homogeneous approximations of the mechanical systems and then successfully extended to the original ones. 


\section{A Some useful inequalities}

In this appendix we prove two lemmas which are used in previous sections.

Lemma A.1 Let $l>1$, then $\forall x, y \in \mathbb{R}:|x-y|^{2} \leq 2^{2(l-1) / l}\left|\{x\}^{\{l\}}-\{y\}^{\{l\}}\right|^{2 / l}$.

Proof. This inequality is true for $x=y$. Assume $(x-y) \neq 0$. For any given $l>1$, let us define for $x, y \in \mathbb{R}(x \neq y) g_{l}(x, y)=\frac{\left|\{x\}^{\{l\}}-\{y\}^{\{l\}}\right|^{2 / l}}{(x-y)^{2}}$. We have $g_{l}(x, y)=h_{l}\left(\frac{y}{x-y}\right)$ with $h_{l}: z \in \mathbb{R} \mapsto$ $\left|\{1+z\}^{\{l\}}-\{z\}^{\{l\}}\right|^{2 / l}$ which is bounded below by $2^{2(1-l) / l}$ which gives the desired inequality.

Lemma A.2 Let $a_{0}>0, a_{1}>0, a_{2}>0$, and $\beta>\gamma>1$, then

$$
\psi(z)=-a_{0}-a_{1} z^{\beta}+a_{2} z^{\gamma}<0, \forall z \in[0,+\infty),
$$

if and only if

$$
a_{0} a_{1}^{\frac{\gamma}{\beta-\gamma}}>a_{2}^{\frac{\beta}{\beta-\gamma}}\left(\left(\frac{\gamma}{\beta}\right)^{\frac{\gamma}{\beta-\gamma}}-\left(\frac{\gamma}{\beta}\right)^{\frac{\beta}{\beta-\gamma}}\right)
$$

Remark A.1 Let $a_{0}>0, a_{1}>0, a_{2}>0, \beta \in \mathbb{R}$, and $\gamma \in \mathbb{R} \backslash\{0\}$. Then

$$
a_{1}>\frac{\gamma}{\beta} \frac{a_{2}^{\frac{\beta}{\gamma}}}{a_{0}^{\frac{\beta-\gamma}{\gamma}}}
$$

implies A.2.

Proof of Lemma A.2. $\psi^{\prime}(z)=-a_{1} \beta z^{\beta-1}+a_{2} \gamma z^{\gamma-1}$ which zeros at 0 and at $z_{0}=\left(\frac{a_{2} \gamma}{a_{1} \beta}\right)^{\frac{1}{\beta-\gamma}}$ thus $\psi$ on $\left[0,+\infty\left[\right.\right.$ has a maximum at $z_{0}: \psi\left(z_{0}\right)=-a_{0}-a_{1}\left(\frac{a_{2} \gamma}{a_{1} \beta}\right)^{\frac{\beta}{\beta-\gamma}}+a_{2}\left(\frac{a_{2} \gamma}{a_{1} \beta}\right)^{\frac{\gamma}{\beta-\gamma}}$ which is negative if and only if A.2 holds.

\section{References}

[1] Fabio Ancona and Alberto Bressan. Flow stability of patchy vector fields and robust feedback stabilization. SIAM J. Control Optim., 41(5):1455-1476, 2002.

[2] Fabio Ancona and Alberto Bressan. Stability rates for patchy vector fields. ESAIM Control Optim. Calc. Var., 10(2):168-200, 2004.

[3] Vincent Andrieu, Laurent Praly, and Alessandro Astolfi. Homogeneous approximation, recursive observer design, and output feedback. SIAM Journal on Control and Optimization, 47(4):1814$1850,2008$.

[4] Emmanuel Bernuau, Wilfrid Perruquetti, Denis Efimov, and Emmanuel Moulay. Finite-time output stabilization of the double integrator. In Proceedings of the 51th IEEE CDC, pages 5906-5911, 2002.

[5] Sanjay P. Bhat and Dennis S. Bernstein. Finite-time stability of continuous autonomous systems. SIAM J. Control Optim., 38(3):751-766, 2000.

[6] Anthony M. Bloch. Nonholonomic Mechanics, pages 207-276. Springer New York, New York, NY, 2003.

[7] Anthony M. Bloch, Mahmut Reyhanoglu, and N. Harris McClamroch. Control and stabilization of nonholonomic dynamic systems. IEEE Transactions on Automatic Control, 37(11):1746-1757, Nov 1992. 
[8] Roger W. Brockett. Asymptotic stability and feedback stabilization. In Differential geometric control theory (Houghton, Mich., 1982), volume 27 of Progr. Math., pages 181-191. Birkhäuser Boston, Boston, MA, 1983.

[9] Guy Campion, Georges Bastin, and Brigitte d'Andréa-Novel. Structural properties and classification of kinematic and dynamic models of wheeled mobile robots. IEEE Transactions on Robotics and Automation, 12(1):47-62, Feb 1996.

[10] Francis H. Clarke, Yuri S. Ledyaev, Ludovic Rifford, and Ronald J. Stern. Feedback stabilization and Lyapunov functions. SIAM J. Control Optim., 39(1):25-48, 2000.

[11] Francis H. Clarke, Yuri S. Ledyaev, Eduardo D. Sontag, and Andrei I. Subbotin. Asymptotic controllability implies feedback stabilization. IEEE Trans. Automat. Control, 42(10):1394-1407, 1997.

[12] Jean-Michel Coron. A necessary condition for feedback stabilization. Systems Control Lett., 14(3):227-232, 1990.

[13] Jean-Michel Coron. Global asymptotic stabilization for controllable systems without drift. Math. Control Signals Systems, 5(3):295-312, 1992.

[14] Jean-Michel Coron. On the stabilization in finite time of locally controllable systems by means of continuous time-varying feedback law. SIAM J. Control Optim., 33(3):804-833, 1995.

[15] Jean-Michel Coron. Control and nonlinearity, volume 136 of Mathematical Surveys and Monographs. American Mathematical Society, Providence, RI, 2007.

[16] Jean-Michel Coron. Stabilization of control systems and nonlinearities. In Proceedings of the 8th International Congress on Industrial and Applied Mathematics, pages 17-40. Higher Ed. Press, Beijing, 2015.

[17] Jean-Michel Coron and Brigitte d'Andréa-Novel. Smooth stabilizing time-varying control laws for a class of nonlinear systems. application to mobile robots. In Michel Fliess, editor, IFAC Nonlinear Control Systems Design (Bordeaux, France, 24-26 June 1992), pages 649-654, 1993.

[18] Jean-Michel Coron and El-Yazid Keraï. Explicit feedbacks stabilizing the attitude of a rigid spacecraft with two control torques. Automatica J. IFAC, 32(5):669-677, 1996.

[19] Jean-Michel Coron and Laurent Praly. Adding an integrator for the stabilization problem. Systems Control Lett., 17(2):89-104, 1991.

[20] Jean-Michel Coron and Ivonne Rivas. Quadratic approximation and time-varying feedback laws. SIAM J. Control Optim., 55(6):3726-3749, 2017.

[21] Jean-Michel Coron and Lionel Rosier. A relation between continuous time-varying and discontinuous feedback stabilization. J. Math. Systems Estim. Control, 4(1):67-84, 1994.

[22] Brigitte d'Andréa Novel and Sylvain Thorel. Control of non holonomic or under-actuated mechanical systems: the examples of the unicycle robot and the slider. ESAIM Control Optim. Calc. Var., 22(4):983-1016, 2016.

[23] Denis Efimov and Wilfrid Perruquetti. On conditions of oscillations and multi-homogeneity. Mathematics of Control, Signals, and Systems, 28(1):3, Dec 2015.

[24] Jean-Philippe Guilleron. Contrôlabilité d'une équation de Korteweg-de Vries et d'un système d'équations paraboliques couplées. Stabilisation en temps fini par des feedbacks instationnaires. $\mathrm{PhD}$ thesis, Université Pierre et Marie Curie, 2016.

[25] Varda Haimo. Finite time controllers. SIAM J. Control Optim., 24(4):760-770, 1986. 
[26] Henry Hermes. Homogeneous coordinates and continuous asymptotically stabilizing feedback controls. In Differential equations (Colorado Springs, CO, 1989), volume 127 of Lecture Notes in Pure and Appl. Math., pages 249-260. Dekker, New York, 1991.

[27] Yiguang Hong. Finite-time stabilization and stabilizability of a class of controllable systems. Systems Control Lett., 46(4):231-236, 2002.

[28] Jose L. Massera. Contributions to stability theory. Annals of Mathematics, 64(1):182-206, 1956.

[29] Frédéric Mazenc, Kristin Pettersen, and Henk Nijmeijer. Global uniform asymptotic stabilization of an underactuated surface vessel. IEEE Trans. Automat. Control, 47(10):1759-1762, 2002 .

[30] Pascal Morin and Claude Samson. Time-varying exponential stabilization of a rigid spacecraft with two control torques. IEEE Trans. Automat. Control, 42(4):528-534, 1997.

[31] Pascal Morin and Claude Samson. Control of nonlinear chained systems: from the RouthHurwitz stability criterion to time-varying exponential stabilizers. IEEE Trans. Automat. Control, 45(1):141-146, 2000.

[32] Pascal Morin, Claude Samson, Jean-Baptiste Pomet, and Zhong-Ping Jiang. Time-varying feedback stabilization of the attitude of a rigid spacecraft with two controls. Systems Control Lett., 25(5):375-385, 1995.

[33] Emmanuel Moulay and Wilfrid Perruquetti. Finite time stability and stabilization of a class of continuous systems. Journal of Mathematical Analysis and Applications, 323(2):1430 - 1443, 2006 .

[34] Richard M. Murray and S. Shankar Sastry. Nonholonomic motion planning: steering using sinusoids. IEEE Transactions on Automatic Control, 38(5):700-716, May 1993.

[35] Kristin Y. Pettersen and Olav Egeland. Exponential stabilization of an underactuated surface vessel. In Proceedings of the 35th IEEE CDC, volume 1, pages 967-972, 1996.

[36] Kristin Y. Pettersen and Olav Egeland. Robust control of an underactuated surface vessel with thruster dynamics. In Proceedings of the 1997 American Control Conference, volume 5, pages 3411-3415, 1997.

[37] Andrey Polyakov. Nonlinear feedback design for fixed-time stabilization of linear control systems. IEEE Transactions on Automatic Control, 57(8):2106-2110, Aug 2012.

[38] Andrey Polyakov, Denis Efimov, and Wilfrid Perruquetti. Finite-time and fixed-time stabilization: Implicit lyapunov function approach. Automatica, 51:332 - 340, 2015.

[39] Jean-Baptiste Pomet. Explicit design of time-varying stabilizing control laws for a class of controllable systems without drift. Systems Control Lett., 18(2):147-158, 1992.

[40] Laurent Praly, Brigitte d'Andréa-Novel, and Jean-Michel Coron. Lyapunov design of stabilizing controllers for cascaded systems. IEEE Trans. Automat. Control, 36(10):1177-1181, 1991.

[41] Christophe Prieur. Asymptotic controllability and robust asymptotic stabilizability. SIAM J. Control Optim., 43(5):1888-1912, 2005.

[42] Christophe Prieur and Alessandro Astolfi. Robust stabilization of chained systems via hybrid control. IEEE Trans. Automat. Control, 48(10):1768-1772, 2003. New directions on nonlinear control.

[43] Christophe Prieur and Emmanuel Trélat. Robust optimal stabilization of the Brockett integrator via a hybrid feedback. Math. Control Signals Systems, 17(3):201-216, 2005. 
[44] Lionel Rosier. Homogeneous Lyapunov function for homogeneous continuous vector fields. Systems Control Lett., 19(6):467-473, 1992.

[45] Claude Samson. Velocity and torque feedback control of a nonholonomic cart. In C. Canudas de Wit, editor, Advanced robot control (Grenoble, 1990), volume 162 of Lecture Notes in Control and Inform. Sci., pages 125-151. Springer, Berlin, 1991.

[46] Claude Samson. Control of chained systems application to path following and time-varying point-stabilization of mobile robots. IEEE Trans. Automat. Control, 40(1):64-77, 1995.

[47] Eduardo D. Sontag. Clocks and insensitivity to small measurement errors. ESAIM Control Optim. Calc. Var., 4:537-557, 1999.

[48] Héctor J. Sussmann. A general theorem on local controllability. SIAM J. Control Optim., 25(1):158-194, 1987. 